Research Article

\title{
Deep Learning Approaches for Detecting Pneumonia in COVID-19 Patients by Analyzing Chest X-Ray Images
}

\author{
M. D. Kamrul Hasan, ${ }^{1}$ Sakil Ahmed ${ }^{1},{ }^{1}$ Z. M. Ekram Abdullah $\left(D,{ }^{1}\right.$ \\ Mohammad Monirujjaman Khan (D), ${ }^{1}$ Divya Anand $\left(D,{ }^{2}\right.$ Aman Singh (D), \\ Mohammad AlZain (D, ${ }^{3}$ and Mehedi Masud (D) ${ }^{4}$ \\ ${ }^{1}$ Department of Electrical and Computer Engineering, North South University, Bashundhara, Dhaka-1229, Bangladesh \\ ${ }^{2}$ Department of Computer Science and Engineering, Lovely Professional University, Punjab-144411, Phagwara, India \\ ${ }^{3}$ Department of Information Technology, College of Computers and Information Technology, Taif University, P. O. Box 11099, \\ Taif 21944, Saudi Arabia \\ ${ }^{4}$ Department of Computer Science, College of Computers and Information Technology, Taif University, P. O. Box 11099, \\ Taif 21944, Saudi Arabia
}

Correspondence should be addressed to Mehedi Masud; mmasud@tu.edu.sa

Received 20 March 2021; Accepted 3 May 2021; Published 19 May 2021

Academic Editor: Kehui Sun

Copyright (c) 2021 M. D. Kamrul Hasan et al. This is an open access article distributed under the Creative Commons Attribution License, which permits unrestricted use, distribution, and reproduction in any medium, provided the original work is properly cited.

\begin{abstract}
The COVID-19 pandemic has wreaked havoc in the daily life of human beings and devastated many economies worldwide, claiming millions of lives so far. Studies on COVID-19 have shown that older adults and people with a history of various medical issues, specifically prior cases of pneumonia, are at a higher risk of developing severe complications from COVID-19. As pneumonia is a common type of infection that spreads in the lungs, doctors usually perform chest X-ray to identify the infected regions of the lungs. In this study, machine learning tools such as LabelBinarizer are used to perform one-hot encoding on the labeled chest X-ray images and transform them into categorical form using Python's to_categorical tool. Subsequently, various deep learning features such as convolutional neural network (CNN), VGG16, AveragePooling2D, dropout, flatten, dense, and input are used to build a detection model. Adam is used as an optimizer, which can be further applied to predict pneumonia in COVID-19 patients. The model predicted pneumonia with an average accuracy of $91.69 \%$, sensitivity of $95.92 \%$, and specificity of $100 \%$. The model also efficiently reduces training loss and increases accuracy.
\end{abstract}

\section{Introduction}

COVID-19, acknowledged by the World Health Organization (WHO) as a pandemic, has dramatically altered the course of humans' daily lives, their immediate health, and economies throughout the world [1]. A rapidly spreading viral disease, COVID-19 affects humans and animals. As per Worldometers, approximately 2,869,886 people have died due to coronavirus complications so far [2]. In most cases, it is pneumonia that makes this disease highly dangerous and potentially fatal. Therefore, detecting and diagnosing pneumonia in COVID-19 patients is critical. The use of deep learning techniques can help us achieve this goal. Deep learning algorithms can be used to detect and diagnose pneumonia using only X-ray images, in the process saving both money and time. Doctors can also benefit from the use of this approach as it can help them efficiently identify highly critical patients so as to isolate them from patients with milder symptoms. In this way, appropriate medical treatments can be administered to COVID-19 patients, which can potentially save many lives.

Many organizations and research institutions are trying to develop vaccines, and there are now several approved and in use. Furthermore, studies on COVID-19 patients have shown that people with a history of lung infections are most severely affected by the virus. Chest X-ray and CT images are 
well-known imaging techniques to diagnose lung-related problems. And considering throat infection and experiencing difficulty while breathing are usually the first major symptoms of COVID-19 [2], these imaging techniques can be utilized in detecting lung complications induced by COVID-19 as well. Moreover, the cost of diagnosing COVID-19 is expensive in some countries [3], and thus, a low-cost method for detecting COVID-19 complications is needed.

In this research, we considered X-ray images for detecting COVID-19 patients [4]. The image dataset contained both healthy and COVID-19 patients. This study primarily focuses on the pretrained VGG16 model for predicting pneumonia using chest $\mathrm{X}$-ray images of coronavirus-infected patients.

Deep learning techniques have been applied in medical diagnoses in the last few years. It has enormous potential for extracting minute features by sampling kernels. To date, various deep learning models have been used in different fields [5-11]. Bar et al. [8] used deep learning models to detect chest pathologies. Razzak et al. [9] discussed various problems in the application of deep learning models in medical image processing. The work considered the chest X-ray scans PA view of COVID-19 and pneumonia patients. After preprocessing and applying data augmentation techniques on the chest $\mathrm{X}$-ray images, we considered a pretrained model, VGG16. We collected 6432 chest X-ray images from Kaggle. We used 5144 images for training and 1288 images for validation. The VGG16 model shows an average accuracy of $91.69 \%$ for detecting pneumonia.

Pneumonia induced by COVID-19 can be diagnosed through genetic and imaging tests. And through a rapid detection mechanism, the spread of COVID-19 can be controlled. Various studies have been conducted to identify different types of diseases by analyzing chest X-ray images, from which some of the major works are highlighted below.

The authors in [12] investigated the performance of a deep learning formula to diagnose heart failure using 952 labeled X-ray images. Two cardiologists in the National Institutes of Health examined the images and identified 260 as "normal" and 378 as "heart failure". The remaining images were discarded. The authors achieved $82 \%$ accuracy in diagnosing heart failure using data augmentation and transfer learning. The authors in [12] used data segmentation and transfer learning to predict heart disease, achieving an accuracy of $82 \%$. In the present study, we deal with detecting pneumonia using deep learning techniques on chest X-ray, achieving an accuracy of $91.69 \%$.

The authors in [13] mainly focused on applying a deep learning technique to classify tuberculosis (TB) using chest $\mathrm{X}$-ray images. The authors used different preprocessing techniques, such as augmentation and segmentation, before applying the proposed deep model images. The dataset consisted of $700 \mathrm{~TB}$ infected and 3500 traditional chest X-ray images. Owing to the advancement of transfer learning approaches, many techniques such as VGG-19, ResNet-50, InceptionV3, ChestNet, and DenseNet201 have been proposed and applied to detect TB cases [13]. The accuracy, precision, sensitivity, $F 1$-score, and specificity in detecting this infectious disease were $97.07 \%, 97.34 \%, 97.07 \%, 97.14$ $\%$, and $97.36 \%$, respectively. In [13], the authors used different transfer learning-based convolutional neural networks (CNNs) to predict TB. This research used a fine-tuned CNN-based VGG16 model to classify chest X-ray images of COVID-19 patients and pneumonia patients and detect COVID-19 cases with pneumonia.

The authors in [14] used a system to observe carcinoma using chest X-ray images. The authors considered the 121-layer CNN model called DenseNet-121. The model used a respiratory organ nodule dataset, achieving $74.43 \pm 6.01 \%$ mean accuracy. Meanwhile, the model obtained $74.96 \pm 9.85 \%$ mean specificity and $74.68 \% \pm 15.33 \%$ mean sensitivity. Grewal [15] used a deep learning technique for brain hemorrhage detection. In the present study, the VGG16 model was used, which loads a set of weights pretrained on ImageNet [16], leaving off the FC layer head. The model also used AveragePooling2D of pool_size $(4,4)$, flatten, dense layer, and dropout of 0.5 , for predicting pneumonia. The accuracy of the model was $91.69 \%$, sensitivity was $95.92 \%$, and specificity was $100 \%$, which makes this model unique from those discussed in [12-14]. In addition to the research discussed, numerous studies have proposed models applying machine learning and deep learning to detect COVID-19 and non-COVID-19 patients by analyzing chest $\mathrm{X}$-rays [17-20]. However, no model can be claimed as a standard because the models use different datasets.

This study proposed a deep learning-based model to predict pneumonia in COVID-19 patients using chest X-ray images. Pneumonia is a virus-related disease and in many cases results in patient's death. COVID-19 patients with pneumonia also face various health-related complications. This research helps to detect pneumonia in COVID-19 patients so that they could be separated from other less severe patients and given appropriate life-saving treatment. Here, some deep learning features such as AveragePooling2D, flatten, dense, ImageDataGenerator, and dropout are used to preprocess the data, and a CNN-based VGG16 model is used to classify chest X-ray images of COVID-19 patients and pneumonia patients and predict COVID-19 patients with pneumonia.

Section 2 describes the methods and materials used to build the CNN-based model. Section 3 presents the results and discussion of the results. Finally, in Section 4, we provide our conclusions.

\section{Method and Methodology}

In the first few sections here, we present a basic block diagram of the system and an overview of this research work through a flowchart. The latter part highlights various deep learning features and models used to accurately detect pneumonia in COVID-19 patients. Figure 1 shows the full system overview of the model design. The model consists of the VGG16 pretrained model, an image data generator to generate images, AveragePooling2D, flatten, dense layers, and dropout layers. We also preprocessed the data and performed data augmentation, before detecting pneumonia. A classification matrix was also generated to evaluate $F 1$ score, precision, and recall and then to calculate accuracy, sensitivity, and specificity. 


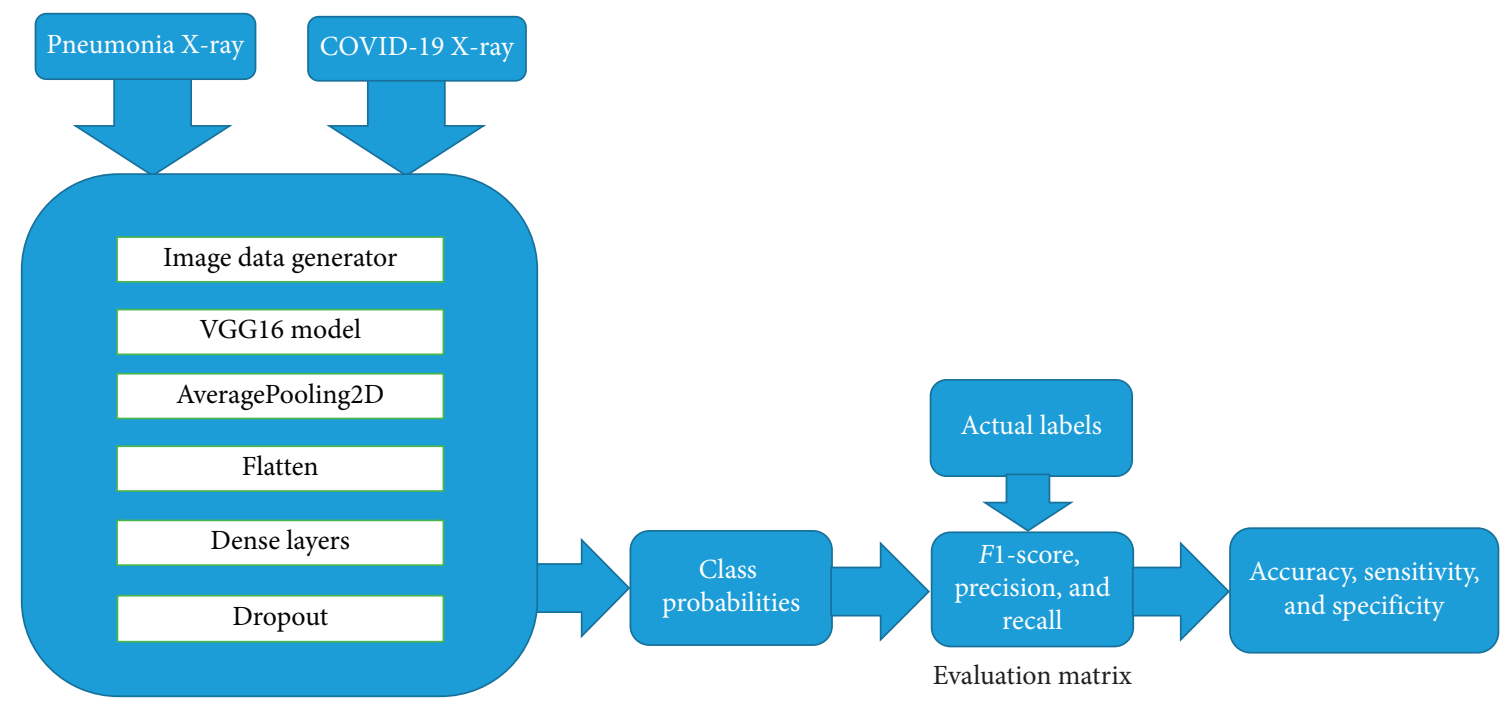

FIGURE 1: Full system block diagram showing the process of how to predict pneumonia in COVID-19 patients.

2.1. Working Steps of Pneumonia Prediction. A deep learning algorithm is used to learn features from patients' $\mathrm{X}$-ray images more accurately so that the model can detect pneumonia more accurately. The following working steps provide complete knowledge of this research work along with the flowchart.

Dataset collection: Kaggle's dataset [4] was used for this research project.

Data processing and augmentation: after collecting images from the dataset, the noise in the X-ray images is removed and cleaned. After that, the data are resized.

Feature extraction: VGG16 model was used to build a pneumonia prediction model.

Data split in training and test set: the data were split into $80 \%$ training and $20 \%$ testing data. Then the data are fed into the VGG16 model for training.

Data test: after training the data into the model, the test data were used for prediction.

Results and conclusions: the final output was then used to calculate accuracy, create a classification matrix, and determine sensitivity and specificity.

A flowchart of this process is shown in Figure 2.

2.2. The Proposed CNN Model. The CNN model used in the present study has two major sections: feature extractors and classifiers. A CNN model uses a hierarchical model that functions to create a network and produces a fully connected layer resembling neurons connected to each other; therefore, this model generates the most efficient results in the classification of images with fewer errors. Figure 3 shows a general CNN architecture used in the present study.

VGG16 is a CNN architecture that is applied to image classification problems on a large image dataset. The model loads pretrained weights on ImageNet, leaving the fully connected (FC) layer head off. The FC layer has three fully connected layers, followed by a series of convolutional layers. There were 4096 channels in each of the first two layers. The third layer has 1000 channels and hence performs ILSVRC classification in 1000 ways. The last layer is the softmax layer, which has the same number of nodes as the output layer. This layer is generally used for multiclass classification problems, where class membership is required for more than two labels.

An epoch in deep learning is a full iteration of the samples. Epoch is a hyperparameter that states the number of iterations a model is applied to the training dataset. In each epoch, the sample in the dataset updates the internal model parameters during training. An epoch may have one or more batches. An epoch with one batch is called the batch gradient descent learning algorithm [22]. In the coding part of this research work, 25 epochs were used.

The hyperparameter batch size defines the number of samples to pass through before updating the model parameters so that each time the model can be improved. The batch size can be considered as iteration over one or more samples to make predictions. The predictions are then compared with the expected output variables at the end of the batch, and the error is calculated. The existing model improves itself from this error, for example, by moving along the error gradient [22]. In the coding part of this study, the batch size was set to 16 and the initial learning rate was $1 e-3$. Flattening is applied for transforming multidimensional data into one-dimensional data to input it into the next layer. In this study, flattening was used to output the convolutional layers to a onedimensional feature vector. The output is then forwarded to the classification layer [23]. We also used an average pooling layer [24] with a pool size of $(4,4)$.

\section{Performance Analysis}

We evaluated the performance of the proposed model based on different metrics: accuracy, recall, sensitivity, specificity, and precision. The metrics are evaluated by various parameters in the confusion matrix, such as true positive (TP), 


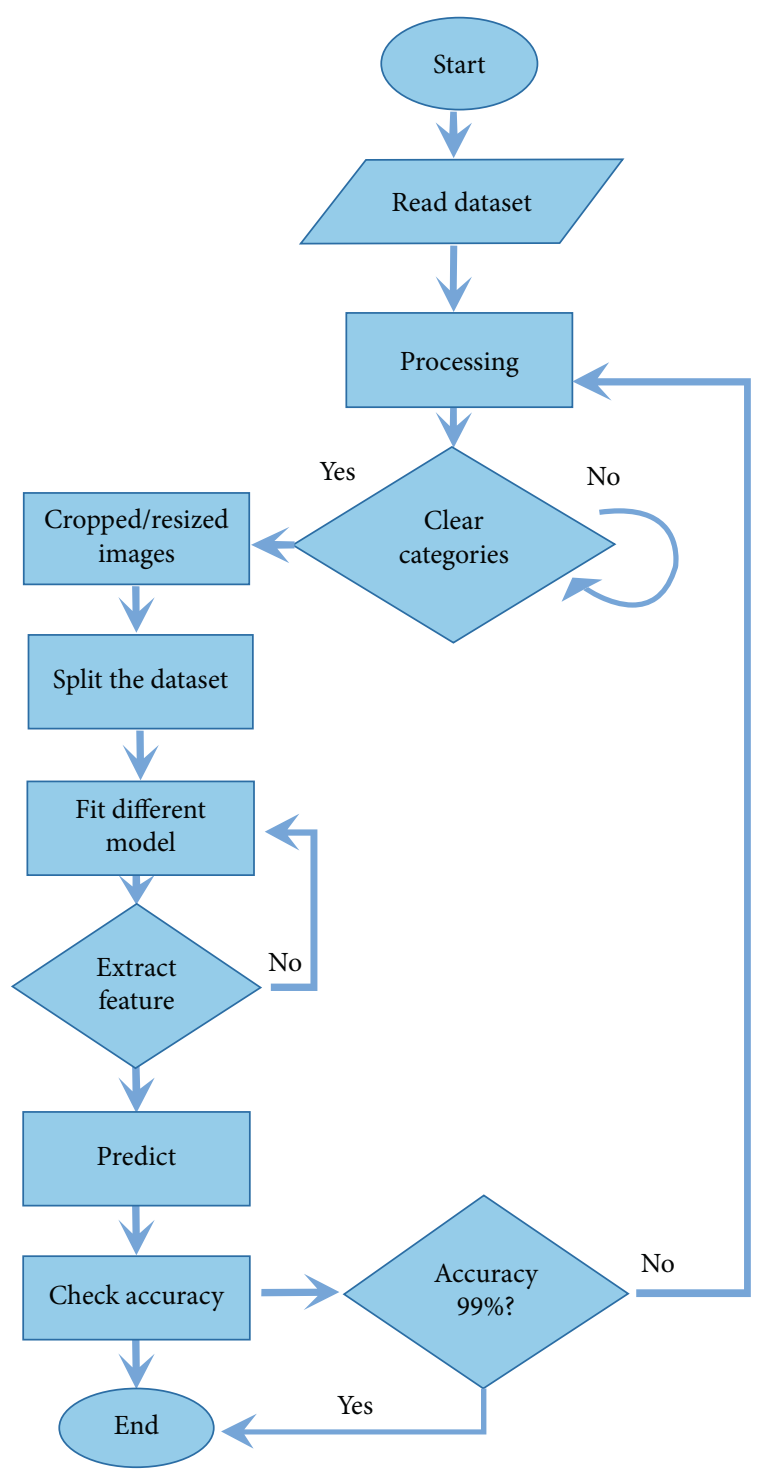

FIGURE 2: Flowchart of the working steps to predict pneumonia in COVID-19 patients.

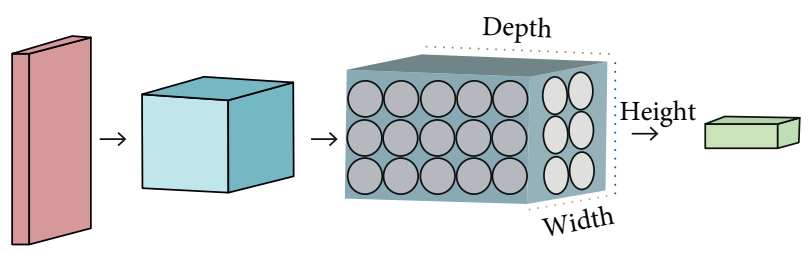

Figure 3: A general 3-dimensional CNN structure [21].

true negative (TN), false positive (FP), and false negative (FN). The metrics are defined as follows:

$$
\text { accuracy }=\frac{\mathrm{TP}+\mathrm{TN}}{\mathrm{TP}+\mathrm{FP}+\mathrm{TN}+\mathrm{FN}}
$$

Sensitivity determines the percentage of actual positive cases that are accurately predicted. This metric evaluates the prediction capability of the model. The equation for calculating the sensitivity is as follows:

$$
\frac{\text { Sensitivity }}{\text { Recall }}=\frac{\mathrm{TP}}{\mathrm{TP}+\mathrm{FN}} \text {. }
$$

To clarify the proportion of actual negative cases, specificity was used, which was predicted correctly. Specificity is a metric that evaluates a model's ability to predict true-negative cases of a given category. Therefore, these metrics were applied to every categorical model to interpret the result. The equation for calculating the specificity is given as follows:

$$
\text { specificity }=\frac{\mathrm{TN}}{\mathrm{FP}+\mathrm{TN}} .
$$

Precision demonstrates the performance of the model on the test data. It shows the number of models predicted correctly from all positive classes. This should be as high as possible:

$$
\text { precision }=\frac{\mathrm{TP}}{\mathrm{TP}+\mathrm{FP}} .
$$

Anaconda's Jupyter notebook was used to build the complete code for predicting pneumonia in COVID-19 patients. Jupyter notebook is an open-source platform, and all the necessary libraries can be accessed to complete this research.

\section{Discussion}

This section discusses the results achieved for predicting pneumonia in COVID-19 patients using the proposed approach. First, we applied data augmentation using the Keras image data generator technique on the images. For the image generator, the rotation angle was kept at 15 and the fill mode was set to nearest. Then, they are applied on LabelBinarizer () to perform one-hot encoding on the labels. After that, the data were split into $80 \%$ training and $20 \%$ testing data, as shown in Table 1. A base model and a head model were built; the head model was transformed using AveragePooling2D [25], flatten, dense, dropout layers, and finally, the complete model was developed. Subsequently, the complete model was compiled with an Adam optimizer, and the testing phase was predicted. However, the accuracy was $91.69 \%$ in predicting pneumonia in COVID-19 patients after formulating chest X-ray images. Table 2 represents various parameters used in the training phase, wherein the deep learning model generates and improves training and validation accuracy after each epoch. After approximately seven epochs, the system achieved the maximum accuracy, where the training loss and validation loss gradually decreased, and the training and validation accuracy increased. In each epoch, the model attains more knowledge after collecting various pieces of information. Therefore, after each epoch, the VGG16 model can provide better accuracy with less training and validation loss.

Table 3 demonstrates that, after training all images from the training dataset, the proposed model starts running epoch after epoch. Although 25 epochs were initially included, after 7 epochs, the model stopped to overcome overfitting. At epoch 1, training accuracy was $89.17 \%$, loss 
TABLE 1: Dataset split for the model's training and testing.

\begin{tabular}{lc}
\hline Dataset & Number of images \\
\hline Training & 5144 \\
Validation & 1288 \\
Testing & 1288 \\
\hline
\end{tabular}

TABLE 2: Model training and validation parameters.

\begin{tabular}{lc}
\hline Parameters & Value \\
\hline Initial learning rate & $1 e-3$ \\
Batch size & 16 \\
Shuffling & Each epoch \\
Optimizer & Adam \\
Max epochs & 25 \\
Execution environment & GPU \\
\hline
\end{tabular}

TABLE 3: Training loss, training accuracy, validation loss, and validation accuracy on different epochs.

\begin{tabular}{ccccc}
\hline & Loss & Accuracy & VAL_Loss & VAL_Accuracy \\
\hline 0 & 0.286430 & 0.891719 & 0.232570 & 0.911491 \\
1 & 0.269631 & 0.900272 & 0.223635 & 0.920031 \\
2 & 0.255112 & 0.903188 & 0.219480 & 0.917702 \\
3 & 0.261968 & 0.905132 & 0.221931 & 0.920807 \\
4 & 0.250308 & 0.904743 & 0.202796 & 0.923137 \\
5 & 0.245776 & 0.905327 & 0.212749 & 0.922360 \\
6 & 0.236960 & 0.910964 & 0.216345 & 0.916925 \\
\hline
\end{tabular}

$28.64 \%$, validation accuracy $91.14 \%$, and validation loss 23.25\%. After seven epochs, training accuracy was $91.1 \%$, loss $23.69 \%$, validation accuracy $91.69 \%$, and validation loss $21.63 \%$.

Figure 4 represents that, after fitting the proposed model for the testing phase, it predicts X-ray images as pneumoniaaffected. To make it clear, the testing phase has been built differently. However, in Figure 5, we show that the model predicts three different classes of chest X-ray images, and the predicted class and actual class are the same. Thus, it can be said that the proposed model could successfully identify each class.

Tables 4 and 5 present the classification report. As can be seen, the precision of COVID-19 prediction is $99 \%$, recall is $81 \%$, and $F 1$-score is $89 \%$, whereas the precision for normal case detection is $83 \%$, recall is $91 \%, F 1$-score is $87 \%$, and the precision for pneumonia prediction is $95 \%$, recall is $93 \%$, and $F 1$-score is $94 \%$. Therefore, it is concluded that using the proposed approach, there are lesser chances of being wrong in diagnosing pneumonia prediction compared with normal and COVID-19 cases.

Figure 6 shows TP and TN pneumonia prediction cases and FP and FN pneumonia prediction cases to make this result clearer: a total of 798 people were found to be affected with pneumonia, and 387 people truly did not have pneumonia. Simultaneously, the model also gives false assumptions: a total of 57 people were falsely diagnosed as having pneumonia and 46 people were falsely diagnosed to not have pneumonia. To address this, this model's sensitivity and

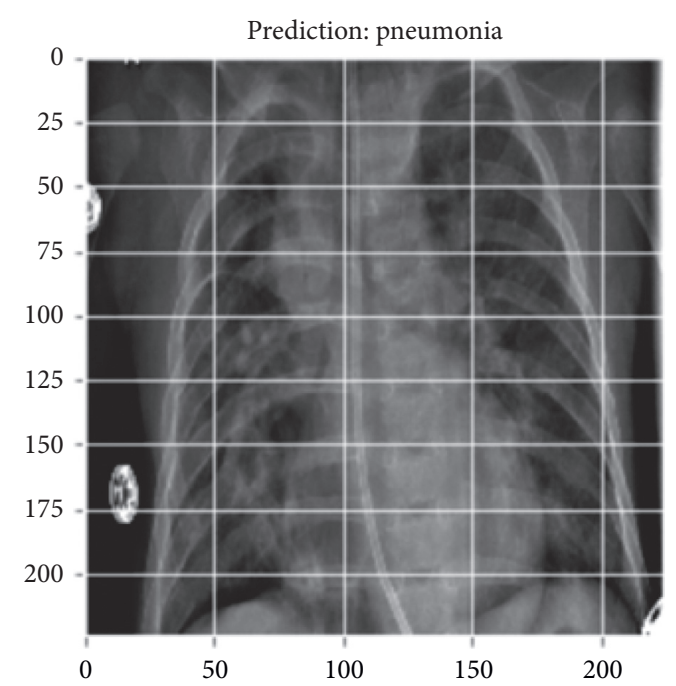

FIgURE 4: Proposed model prediction on a test image.

specificity values are calculated, which are $95.92 \%$ and $100 \%$, respectively. The average accuracy of the proposed method after completing epochs is 91.69, which demonstrates that the model performs well in the classification of COVID-19-induced pneumonia.

Figures 7 and 8 show the accuracy and loss values during the model's training and validation phases. The figures show how training and validation losses decrease and increase training accuracy and validation accuracy. The $x$-axis represents the number of epochs and the $y$-axis represents loss/ accuracy. When the epoch was 0 , both the training and validation accuracies were low, and the loss was very high. But as the number of epochs increased, both training accuracy and validation accuracy increased, and training and validation loss decreased. After seven epochs, the training and validation accuracies are closer to $92 \%$, and the training loss and validation loss are very close to $2 \%$.

Therefore, using the proposed model, the patients may not need to visit a physician and spend large sums of clinical tests and examinations; rather, they only need a chest X-ray and a supporting mobile application to detect pneumonia [25]. Thus, underprivileged and poorer or rural sections of the society will be highly benefitted from the results of the present study. After diagnosing pneumonia through the app, they can simply follow the doctor's guidelines and take appropriate medicines.

The progress made in applying deep learning-based methods using chest X-rays for COVID-19 classification is tremendous. The researchers mainly focused on the detection of three classes: COVID-19, normal, and pneumonia. The scarcity of large datasets is a significant problem in the evaluation of the proposed models. To solve the problem of small-sized datasets, transfer learning methods have been applied [19, 20, 26-30]. The models are pretrained on the ImageNet dataset [16]. Ensemble learning techniques $[18,31,32]$, which combine predictions from several models to produce accurate results, are also used in COVID-19 detection. This enhances the results of the model prediction by minimizing the generalization error and variance. 


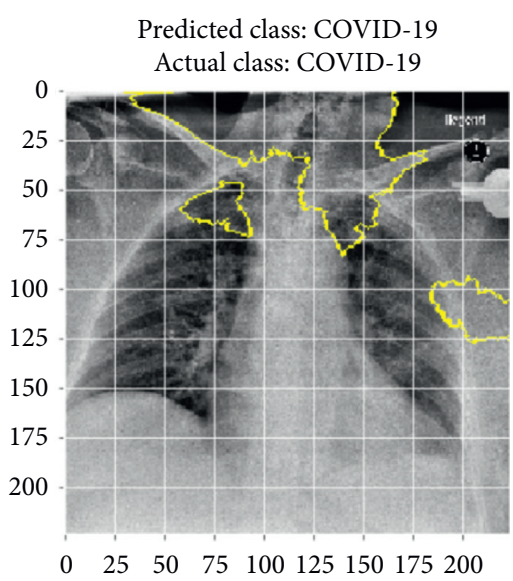

(a)

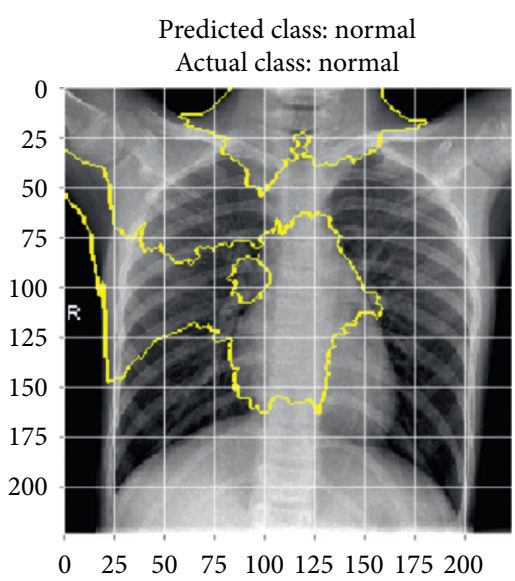

(b)

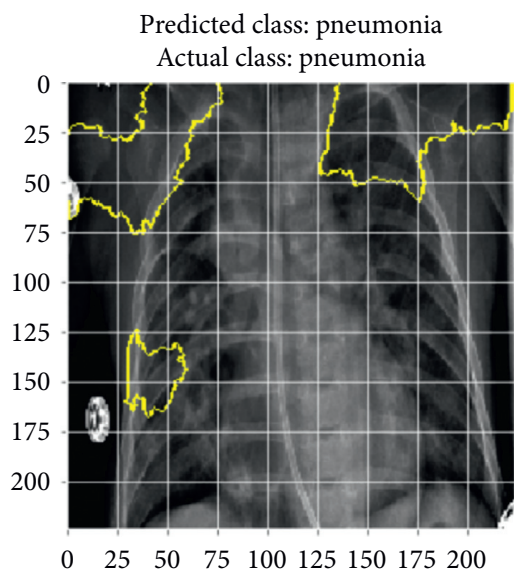

(c)

Figure 5: Actual class vs. predicted class.

TABle 4: Classification report of the pneumonia prediction model.

\begin{tabular}{lcccc}
\hline & Precision & Recall & F1-score & Support \\
\hline 0 & 0.99 & 0.81 & 0.89 & 116 \\
1 & 0.83 & 0.91 & 0.87 & 317 \\
2 & 0.95 & 0.93 & 0.94 & 855 \\
Accuracy & & & 0.92 & 1288 \\
Macro avg & 0.92 & 0.89 & 0.90 & 1288 \\
Weighted avg & 0.92 & 0.92 & 0.92 & 1288 \\
\hline
\end{tabular}

TABle 5: Performance score on test data.

\begin{tabular}{lccc}
\hline Disease & Precision (\%) & Recall (\%) & F1-score (\%) \\
\hline COVID-19 & 99 & 81 & 89 \\
Normal & 83 & 91 & 87 \\
Pneumonia & 95 & 93 & 94 \\
\hline
\end{tabular}

Domain adaptation is another approach that has been used to detect COVID-19 patients using chest X-ray images. Zhang et al. [33] applied this technique to manage data by applying the adaption of feature adversarial and a new classifier approach. The method showed significantly better

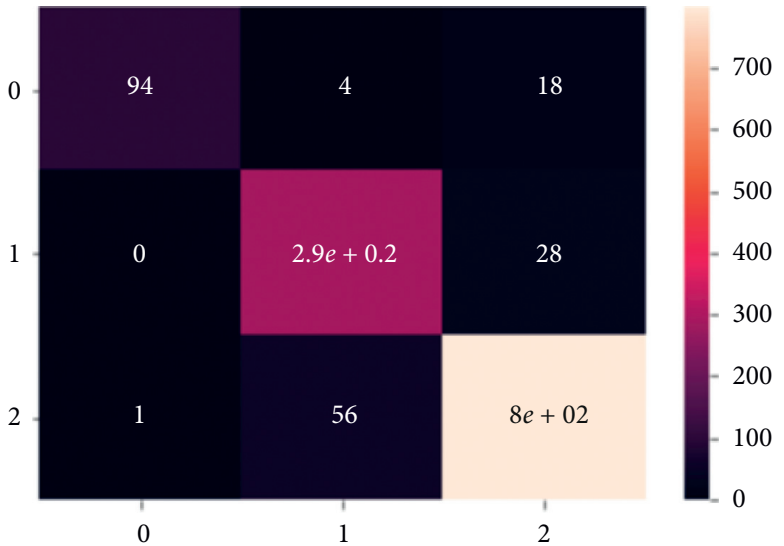

FIgURE 6: Confusion matrix of the pneumonia prediction model.

results in detecting COVID-19. Radiography-based COVID-19 detection suffers from data scarcity. A cascaded network was also introduced for detecting COVID-19. LV et al. [34] cascaded two networks (ResNet-50 and DenseNet169) to detect COVID-19. The authors in [35] proposed a 


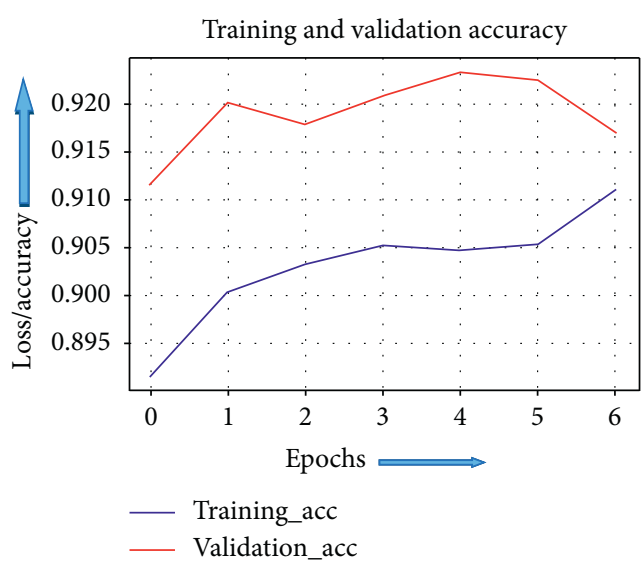

FIgURE 7: Training and validation accuracy graph.

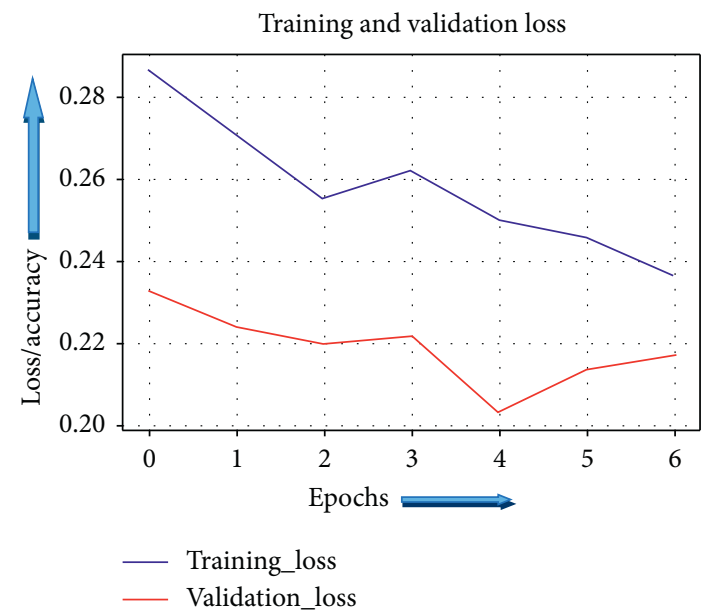

Figure 8: Training and validation loss graph.

game-theoretic model to maintain social distancing to prevent the COVID-19 outbreak in a noncooperative situation. The authors in [36] proposed a security protocol for remote patient care systems using physically unclonable functions to enable doctors to continuously monitor and diagnose COVID-19 patients.

\section{Conclusion}

This research suggests a two-stage deep residual learning technique using lung X-ray images to identify COVID-19induced pneumonia. The model showed good performance in differentiating COVID-19 patients and patients with COVID-19-induced pneumonia using the VGG16 model. The model predicted pneumonia with an average accuracy of $91.69 \%$, sensitivity of $95.92 \%$, and specificity of $100 \%$. It also reduces training loss and increases accuracy. Parallel testing can be used in the current scenario to prevent infection spread to frontline workers and generate primary diagnoses to determine whether a patient is affected by COVID-19. Therefore, the proposed method can be used as an alternative diagnostic tool for detecting pneumonia cases. Future research can improve the $\mathrm{CNN}$ architecture performance by adjusting the hyperparameters and transfer learning combinations. Another feasible way to determine the best model for pneumonia and COVID-19 could be an improved, complex network structure.

\section{Data Availability}

The data used to support the findings of this study are freely available at https://www.kaggle.com/prashant268/chestxray-covid19-pneumonia.

\section{Conflicts of Interest}

The authors declare that they have no conflicts of interest to report regarding this study.

\section{Acknowledgments}

The authors would like to thank the Department of Electrical and Computer Engineering of North South University and Taif University Researchers Supporting Project number (TURSP-2020/98), Taif University, Taif, Saudi Arabia. The authors would like to thank Editage (http://www.editage. com) for English language editing.

\section{References}

[1] O. Gozes, M. Frid-Adar, H. Greenspan, P. D. Browning, H. Zhang et al., "Rapid Ai development cycle for the coronavirus (covid-19) pandemic: initial results for automated detection patient monitoring using deep learning ct image analysis," 2003, https://arxiv.org/abs/2003.05037.

[2] Worldometers,(2020).Coronavirus.: https://www.worldometers. info/coronavirus/?utm_campaign=homeAdvegas 1 ?\%22.

[3] D. M. Hansell, A. A. Bankier, H. MacMahon, T. C. McLoud, N. L. Müller, and J. Remy, "Fleischner society: glossary of terms for thoracic imaging," Radiology, vol. 246, no. 3, pp. 697-722, 2008.

[4] A. Jaiswal, N. Gianchandani, D. Singh, V. Kumar, and M. Kaur, "Classification of the COVID-19 infected patients using densenet201 based deep transfer learning," Journal of Biomolecular Structure and Dynamics, vol. 23, pp. 1-8, 2020.

[5] C. Douarre, R. Schielein, C. Frindel, S. Gerth, and D. Rousseau, "Transfer learning from synthetic data applied to soil-root segmentation in X-ray tomography images," Journal of Imaging, vol. 4, no. 5, p. 65, 2018.

[6] Y. Zhang, G. Wang, M. Li, and S. Han, "Automated classification analysis of geological structures based on images data and deep learning model," Applied Sciences, vol. 8, no. 12, p. 2493, 2018.

[7] Z. Chen, Y. Zhang, C. Ouyang, F. Zhang, and J. Ma, “Automated landslides detection for mountain cities using multitemporal remote sensing imagery," Sensors, vol. 18, p. 821, 2018.

[8] Y. Bar, I. Diamant, L. Wolf, S. Lieberman, E. Konen, and H. Greenspan, "Chest pathology detection using deep learning with non-medical training," in Proceedings of the 2015 IEEE 12th International Symposium on Biomedical Imaging (ISBI), pp. 294-297, New York, NY, USA, April 2015.

[9] M. I. Razzak, S. Naz, and A. Zaib, "Deep learning for medical image processing: overview, challenges and the future," Lecture Notes in Computational Vision and Biomechanics, vol. 32, pp. 323-350, 2018. 
[10] A. Narin, C. Kaya, and Z. Pamuk, "Automatic detection of coronavirus disease (covid-19) using $\mathrm{X}$-ray images and deep convolutional neural networks," 2020, https://arxiv.org/abs/ 2003.10849.

[11] V. V. I. S. S. J. Szegedy, "Rethinking the inception architecture for computer vision," in Proceedings of the IEEE Conference on Computer Vision and Pattern Recognition, pp. 2818-2826, Las Vegas, NV, USA, June 2016.

[12] A. Sharma, S. Rani, and D. Gupta, "Artificial intelligencebased classification of chest X-ray images into COVID-19 and other infectious diseases," International Journal of Biomedical Imaging, vol. 2020, Article ID 8889023, 5 pages, 2020.

[13] Researchgate.https://www.researchgate.net/publication/ 343030475_Diagnosing_Heart_Failure_from_Chest_X-Ray_ Images_Using_Deep_Learning.

[14] X. Li, Y. Zhuang, and S. X. Yang, "Cloud computing for big data processing," Intelligent Automation \& Soft Computing, vol. 23, no. 4, pp. 545-546, 2017.

[15] M. Grewal, M. M. Srivastava, P. Kumar, and S. Varadarajan, "Radnet: radiologist level accuracy using deep learning for hemorrhage detection in ct scans," in Proceedings of the 2018 IEEE 15th International Symposium on Biomedical Imaging, pp. 281-284, Washington, DC, USA, April 2018.

[16] J. Deng, W. Dong, R. Socher, L. J. Li, K. Li, and L. Fei-Fei, "Imagenet: a large-scale hierarchical image database," in Proceedings of the 2009 IEEE Conference on Computer Vision and Pattern Recognition, pp. 248-255, Miami, FL, USA, June 2009.

[17] M. Kaur, V. Kumar, V. Yadav, D. Singh, N. Kumar, and N. N. Das, "Metaheuristic-based deep COVID-19 screening model from chest X-ray images," Journal of Healthcare Engineering, vol. 2020, Article ID 8829829, 17 pages, 2021.

[18] N. Gianchandani, A. Jaiswal, D. Singh et al., "Rapid COVID19 diagnosis using ensemble deep transfer learning models from chest radiographic images," Journal of Ambient Intelligence and Humanized Computing, vol. 12, 2020.

[19] N. Narayan Das, N. Kumar, M. Kaur, V. Kumar, and D. Singh, "Automated deep transfer learning-based approach for detection of COVID-19 infection in chest X-rays," in IRBMElsevier, Amsterdam, Netherlands, 2020.

[20] https://www.kaggle.com/prashant268/chest-xray-covid19pneumonia.

[21] Missinglink,(2019). https://missinglink.ai/guides/neural-networkconcepts/convolutiona;-neural-network-build-one-keraspytorch/.

[22] Ü. H. Ayan, "Diagnosis of pneumonia from chest X-ray images using deep learning," in Proceedings of the 2019 Scientific Meeting on Electrical-Electronics \& Biomedical Engineering and Computer Science (EBBT), pp. 1-5, Istanbul, Turkey, April 2019.

[23] J. Brownlee, Difference between a Batch and an Epoch in a Neural Network, Machine Learning Mastery, San Francisco, CL, USA, 2021, https://machinelearningmastery.com/ difference-between-a-batch-and-an-epoch/.

[24] Towardsdatascience, "The most intuitive and eauest guide for CNN," 2020, https://towardsdatascience.com/the-most-intuitiv e-and-easiest-guide-for-convolutional-neuralnetwork360 7be 47480 :\%7E:text=Flattening\%20is\%20 converting $\% 20$ the $\% 20$ data,called $\% 20$ a $\% 20$ fully $\% 2$ Dconnected $\% 20$ layer.

[25] F. Keras, "Pooling layers," 2020, https://keras.io/api/layers/ pooling_layers/average_pooling2d/.

[26] S. Minaee, R. Kafieh, M. Sonka, S. Yazdani, and G. J. Soufi, "Deep-covid: predicting covid-19 from chest X-ray images using deep transfer learning," 2020, https://arxiv.org/abs/ 2004.09363.

[27] S. Misra, S. Jeon, S. Lee, R. Managuli, and C. Kim, "Multichannel transfer learning of chest X-ray images for screening of COVID-19,” 2020, https://arxiv.org/abs/2005.05576.

[28] F. Ucar and D. Korkmaz, "COVIDiagnosis-Net: deep bayessqueezenet based diagnosis of the coronavirus disease 2019 (COVID-19) from X-ray images," Medical Hypotheses, vol. 140, Article ID 109761, 2020.

[29] M. M. A. Asmaa Abbas, "Classification of COVID-19 in chest $\mathrm{X}$-ray images using DeTraC deep convolutional neural network," Artificial Intelligence Applications for COVID-19, Detection, Control, Prediction, and Diagnosis, vol. 33, 2020.

[30] M. Shorfuzzaman and M. Masud, "On the detection of covid19 from chest x-ray images using cnn-based transfer learning," Computers, Materials \& Continua, vol. 64, no. 3, pp. 1359-1381, 2020.

[31] B. D. Goodwin, C. Jaskolski, C. Zhong, and H. Asmani, "Intramodel variability in COVID-19 classification using chest X-ray images," 2020, https://arxiv.org/abs/2005.02167.

[32] M. Karim, T. Döhmen, D. Rebholz-Schuhmann et al., "Deepcovidexplainer: explainable COVID-19 predictions based on chest X-Ray images," 2020, https://arxiv.org/abs/ 2004.04582.

[33] Y. Zhang, S. Niu, Z. Qiu et al., "COVID-DA: deep domain adaptation from typical pneumonia to COVID-19," 2020, https://arxiv.org/abs/2005.01577.

[34] D. Lv, W. Qi, Y. Li, L. Sun, and Y. Wang, "A cascade network for detecting COVID-19 using chest X-Rays,” 2020, https:// arxiv.org/abs/2005.01468.

[35] A. K. Bairagi, M. Masud, D. H. Kim et al., "Controlling the outbreak of COVID-19: a noncooperative game perspective," IEEE Access, vol. 8, pp. 215570-215581, 2020.

[36] M. Masud, G. S. Gaba, S. Alqahtani et al., "A lightweight and robust secure key establishment protocol for internet of medical things in COVID-19 patients care," IEEE Internet of Things Journal, vol. 12, 2021. 